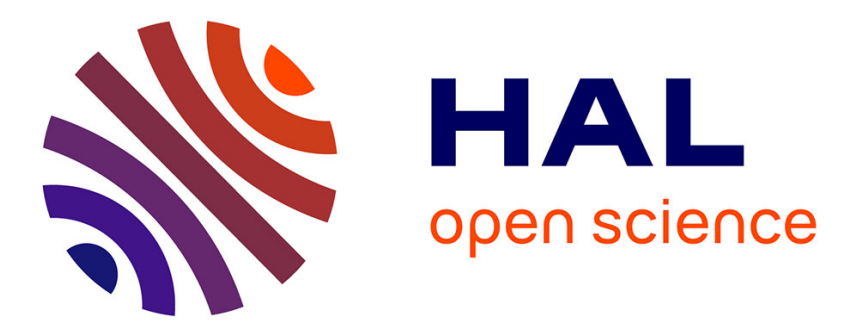

\title{
Symmetry degeneration and room temperature ferroelectricity in ion-irradiated $\mathrm{SrTiO} 3$
}

F Zhang, Haizhou Xue, J Keum, Alexandre Boulle, Yanwen Zhang, W Weber

\section{To cite this version:}

F Zhang, Haizhou Xue, J Keum, Alexandre Boulle, Yanwen Zhang, et al.. Symmetry degeneration and room temperature ferroelectricity in ion-irradiated SrTiO 3. Journal of Physics: Condensed Matter, 2020, 32 (35), pp.355405. 10.1088/1361-648X/ab8ec7 . hal-03034471

\section{HAL Id: hal-03034471 https://hal.science/hal-03034471}

Submitted on 3 Dec 2020

HAL is a multi-disciplinary open access archive for the deposit and dissemination of scientific research documents, whether they are published or not. The documents may come from teaching and research institutions in France or abroad, or from public or private research centers.
L'archive ouverte pluridisciplinaire $\mathbf{H A L}$, est destinée au dépôt et à la diffusion de documents scientifiques de niveau recherche, publiés ou non, émanant des établissements d'enseignement et de recherche français ou étrangers, des laboratoires publics ou privés. 


\title{
Symmetry degeneration and room temperature ferroelectricity in ion irradiated $\mathrm{SrTiO3}$
}

\author{
F X Zhang ${ }^{1}$, Haizhou Xue ${ }^{2}$, J K Keum ${ }^{3}$, A Boulle ${ }^{4}$, Y Zhang ${ }^{1,2}$,WJ Weber ${ }^{1,2}$ \\ ${ }^{1}$ Materials Science and Technology Division, Oak Ridge National Laboratory, Oak Ridge, TN 37831, \\ United States of America \\ ${ }^{2}$ Department of Materials Science and Engineering, University of Tennessee, Knoxville, TN 37996, \\ United States of America \\ ${ }^{3}$ Center for Nanophase Materials Sciences, Oak Ridge National Laboratory, Oak Ridge, TN 37831, \\ United States of America \\ ${ }^{4}$ Institut de Recherche sur les Céramiques, CNRS UMR 7315, Centre Européen de la Céramique, 12 rue \\ Atlantis, 87068 Limoges Cedex, France
}

\begin{abstract}
Polar phonon modes associated with room temperature ferroelectricity are observed in $\mathrm{SrTiO}_{3}$ single crystals irradiated with Ti ions. Quantitative strain analysis reveals that irradiation-induced out-of-plane strain drives the centrosymmetric cubic $\mathrm{SrTiO}_{3}$ to a tetragonal-like structure in the maximum damaged region. Energy transfer from ions to electrons during ion irradiation yields defects in $\mathrm{SrTiO}_{3}$ that also plays an important role for the room temperature ferroelectricity. Different from thin $\mathrm{f} 1 \mathrm{~m}$ techniques, the ferroelectricity in the ion irradiated $\mathrm{SrTiO}_{3}$ can occur for much larger thicknesses, depending on the energy and type of ion.
\end{abstract}

\section{Introduction}

Bulk $\mathrm{SrTiO}_{3}$ has a centrosymmetric cubic structure at room temperature, and there is a cubic to tetragonal structural transition at $105 \mathrm{~K}$ [1]. The dielectric constant of $\mathrm{SrTiO}_{3}$ deviates from the classical Curie-Weiss law at low temperature, and it increases rapidly (up to $1.8 \times 10^{4}$ at $1.4 \mathrm{~K}$ ) as the temperature is reduced [2]. The low temperature electric behavior of $\mathrm{SrTiO}_{3}$ approaches a ferroelectric phase transition; however, bulk $\mathrm{SrTiO}_{3}$ is still paraelectric to the lowest temperature as a result of quantum f uctuations [3]. The 'quantum paraelectrics' or 'incipient ferroelectronics' of $\mathrm{SrTiO}_{3}$ and other perovskite oxides have been a topic of considerable interest during the past few decades [3-5].
The ferroelectric transition of $\mathrm{SrTiO}_{3}$ at low temperature can be induced by strains from lattice mismatch $[5,6]$, chemical $[7,8]$ or isotopic [9] substitution, electric f eld [10, 11], pressure [14] and controlled grain size [12]. Due to important applications in electronic devices, the coupling between strain and ferroelectricity in $\mathrm{SrTiO}_{3} \mathrm{flms}$ has been intensively studied, and the relevant work is well illustrated in a recent review article [13]. By controlling the in-plane lattice strain, the critical temperature $T_{\mathrm{c}}$ of ferroelectric transition in thin $\mathrm{f} / \mathrm{m} \mathrm{SrTiO}_{3}$ or superlattice can be profoundly enhanced [6, 14-18], even to room temperature [5]. However, the biaxial strains in thin $\mathrm{f} / \mathrm{ms}$ depend on the choice of substrate and $\mathrm{f} \mathrm{lm}$ thickness, which turns out to be challenging for the synthesis of uniformly strained $\mathrm{f} 1 \mathrm{~ms}$ because of the undesirable relaxation that occur when the thickness of a sample exceeds the 
critical value. Room temperature ferroelectricity has also been reported for nonstoichiometric $\mathrm{SrTiO}_{3}$ f lms with controlled $\mathrm{Sr}$ or $\mathrm{O}$ def ciencies $[19,22]$, though the mechanism is still under debate $[15,20,21]$.

One fundamental property of ferroelectrics that changes quantitatively during phase transition is the dynamics of lattice vibration. The lattice dynamics of bulk $\mathrm{SrTiO}_{3}$ has been studied theoretically and experimentally with neutron scattering techniques decades ago [22]. As a powerful tool to study the lattice properties, Raman scattering has been successfully used to detect the symmetry degeneration and phase transition in $\mathrm{SrTiO}_{3}$. Since all the zone-center optical phonons of the ideal cubic $\mathrm{SrTiO}_{3}$ are of odd parity, no f rst-order Raman scattering is expected to occur at ambient conditions. Instead, broad second-order peaks are observed in the wavelength ranges of $200-500 \mathrm{~cm}^{-1}$ and $550-750 \mathrm{~cm}^{-1}$. Using an ultraviolet Raman spectroscopy technique, nanoscale ferroelectricity was successfully probed in ultrathin $\mathrm{flms}$ of $\mathrm{BaTiO}_{3} / \mathrm{SrTiO}_{3}$ superlattice [23]. In the present work, we studied the response of ho01i-oriented $\mathrm{SrTiO}_{3}$ single crystals to ion irradiation with Raman scattering and $\mathrm{x}$-ray diffraction techniques. The appearance of forbidden optical phonon modes in the damaged regions suggests room temperature ferroelectricity in the ion-irradiated $\mathrm{SrTiO}_{3}$. The strain depth prof le in ion irradiated $\mathrm{SrTiO}_{3}$ is quantitatively analyzed by single crystal x-ray diffraction measurements, and the results indicate a large tetragonal distortion with a signif cant out-of-plane lattice strain $(1.1-1.3 \%)$ and negligible in-plane strain. The tetragonal distortion promotes the ferroelectric transition of ion irradiated $\mathrm{SrTiO}_{3}$. The defects in the lattice induced by the energy transfer from $\mathrm{Ti}$ ions to the electronic structure also plays an important role for the ferroelectric transition in $\mathrm{SrTiO}_{3}$ crystals.

\section{Experimental procedure}

The ho01i-oriented single crystals of $\mathrm{SrTiO}_{3}$, with size of 12 $\times 15 \mathrm{~mm}^{2}$, were irradiated at room temperature with $12 \mathrm{MeV}$ or $20 \mathrm{MeV}$ Ti ions to fuences from $1 \times 10^{12}$ to $1 \times 10^{14}$ $\mathrm{cm}^{-2}$, with multiple irradiated areas of $3 \times 3 \mathrm{~mm}^{2}$ per sample. The ion irradiations were performed at several degrees off the surface normal to avoid channeling effects using the capabilities of the Ion Beam Materials Laboratory at the University of Tennessee [24]. The Raman spectra from the $\mathrm{SrTiO}_{3}$ were collected in a ref ection conf guration using a high-resolution Raman spectrometer (LabRaman HR Evolution) at room temperature with a $532 \mathrm{~nm}$ green laser as the activation light. Irradiation-induced strain along the direction normal to the h001i-oriented surface was measured with a high-resolution thin $\mathrm{flm} x$-ray diffractometer (PANalytical X'pert Pro) in $2 \theta-\theta$ scan mode with $\mathrm{Cu} K \alpha \mathrm{l}$-ray radiation $(\lambda=1.5406$ $\AA)$. The strain prof les for samples irradiated with $12 \mathrm{MeV}$ Ti ions were derived from simulation of the XRD patterns with the program RaDMaX [25]. Reciprocal space maps were also recorded for the symmetric (002) and asymmetric (103) lattice planes of the samples irradiated with both $12 \mathrm{MeV}$ and $20 \mathrm{MeV}$ Ti ions, and the latter being used to determine the in-plane lattice strain.

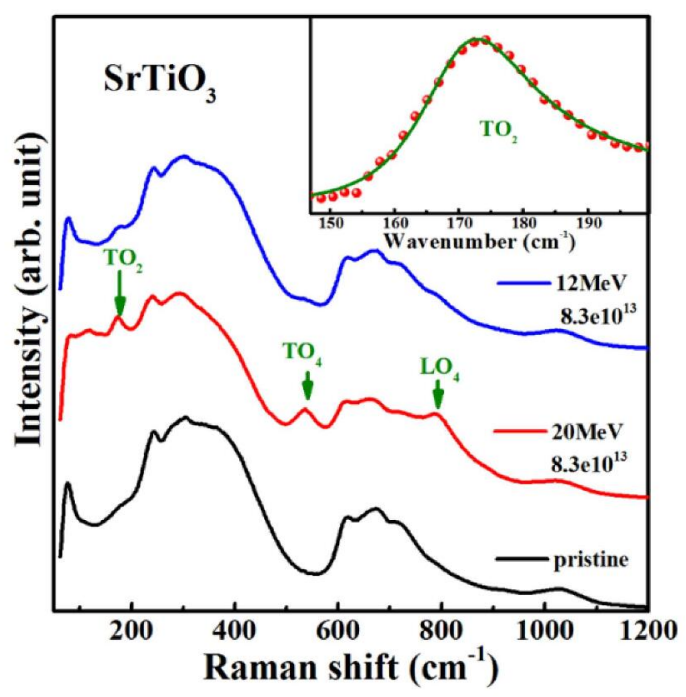

Figure 1 (a) The observed Raman spectrum of pristine $\mathrm{SrTiO}_{3}$ and damaged with $\mathrm{Ti}$ ions of $12 \mathrm{MeV}$ and $20 \mathrm{MeV}$ in $\mathrm{f}$ uence of $8.3 \times 10^{13}$ ions $\mathrm{cm}^{-2}$. (b) The $\mathrm{ftting}$ of the Fano asymmetry Raman modes $\mathrm{TO}_{2}$ and $\mathrm{TO}_{4}$ for the $20 \mathrm{MeV}$ Ti ions irradiated sample. The Fano f tting parameters are $A=39.15, q=4.11, \omega_{b}=170.2 \mathrm{~cm}^{-1}$, and $\Gamma=21.98 \mathrm{~cm}^{-1}$

\section{Results and discussion}

All the irradiated spots were characterized by Raman scattering measurements. There is only second-order Raman scattering signal in pristine $\mathrm{SrTiO}_{3}$ because of the centrosymmetric structure. We observed additional Raman active modes in the irradiated samples, especially for the $20 \mathrm{MeV}$ Ti ion irradiated spots. Figure 1 shows the Raman spectrum of $\mathrm{SrTiO}_{3}$ irradiated with $12 \mathrm{MeV}$ and $20 \mathrm{MeV}$ Ti ions to a fuence of $8.3 \times$ $10^{13} \mathrm{~cm}^{-2}$, together with the spectrum from the pristine sample. The additional modes appearing in the sample irradiated with $20 \mathrm{MeV}$ Ti ions are the polar transverse optic modes $\mathrm{TO}_{2}$, $\mathrm{TO}_{4}$ and polar longitudinal $\mathrm{LO}_{4}$ modes, which are centered at $\sim 170,550$ and $800 \mathrm{~cm}^{-1}$, respectively. The appearance of these polar phonon modes in the irradiated samples is similar to the modes observed in ferroelectric flms before [23, $26,27]$, which is an indication of ferroelectricity. The corresponding phonon modes in the $12 \mathrm{MeV}$ irradiated $\mathrm{SrTiO}_{3}$ are weaker. In the $20 \mathrm{MeV}$ Ti ion irradiated sample, there is a weak peak centered at $\sim 140 \mathrm{~cm}^{-1}$, which is the $\mathrm{e}_{\mathrm{g}}$ mode of tetragonal $\mathrm{SrTiO}_{3}$ [28]. Similar to the Raman spectrum of strained $\mathrm{SrTiO}_{3}$ thin $\mathrm{flms}$, the appearance of the frst-order Raman modes is due to breaking of the symmetry after ion irradiation. The ferroelectricity is caused by the polarization displacement of cations and anions in the damaged region. As observed in thin flms [18] and nano-structured $\mathrm{SrTiO}_{3}$ [12], a pronounced Fano asymmetric peak shape is seen for the $\mathrm{TO}_{2}$ mode, which suggests coherent interference between the discrete phonon and a broad peak [29]. The Fano $\mathrm{TO}_{2}$ mode in ion irradiated $\mathrm{SrTiO}_{3}$ single crystal can also be $\mathrm{ftted}$ by the formula

$$
I(\omega)=A \frac{[q+E(\omega)]^{2}}{1+E(\omega)^{2}}
$$




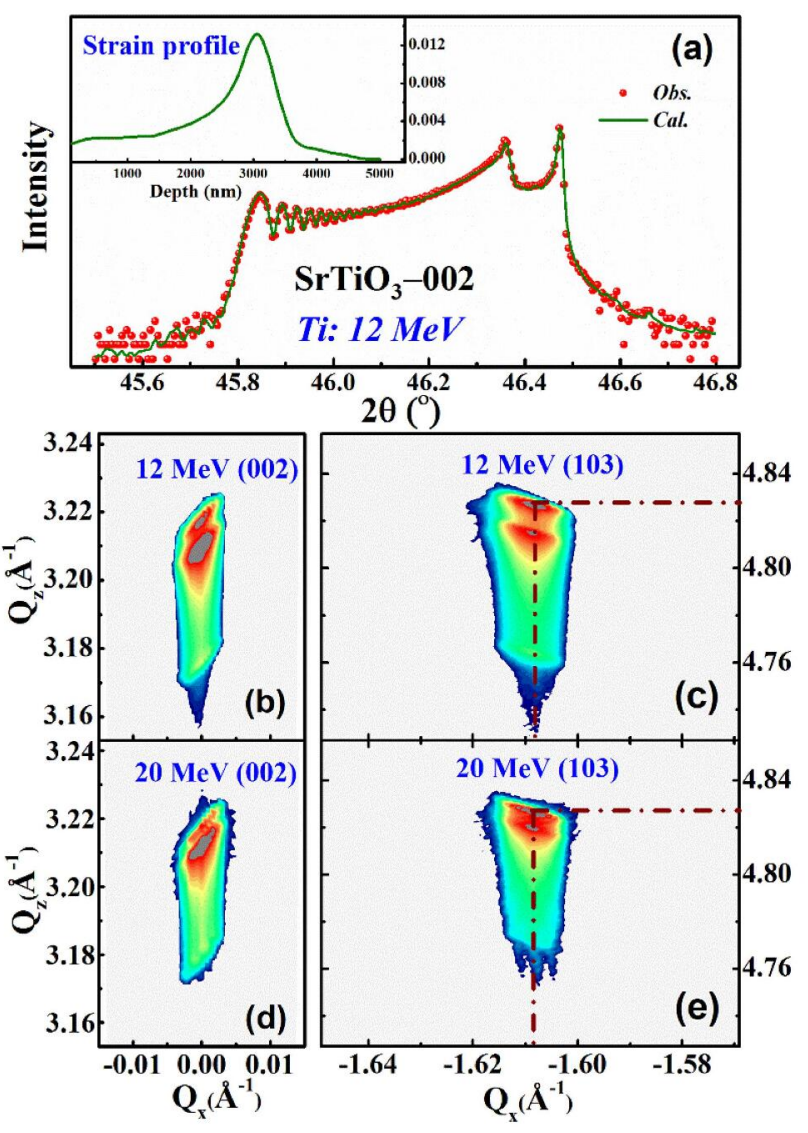

Figure 2. (a) The observed and calculated XRD prof les of the 12 $\mathrm{MeV}$ Ti ion irradiated $\mathrm{SrTiO}_{3}$ around the Bragg peak (002). The maximum strain is $\sim 1.32 \%$, and the corresponding depth dependent strain prof les are shown as inserts; (b) and (c) the reciprocal space maps of $12 \mathrm{MeV}$ Ti ion irradiated sample around the $(002)$ and (103) Bragg peaks; (d) and (e) the reciprocal space maps of $20 \mathrm{MeV}$ $\mathrm{Ti}$ ion irradiated $\mathrm{SrTiO}_{3}$ near the lattice point of $(002)$ and (103).

after subtracting a constant base line, where $E(\boldsymbol{\omega})=2(\boldsymbol{\omega}-$ $\left.\omega_{0}\right) / \Gamma$, and $\omega_{0}$ is the phonon frequency, $A$ is the amplitude, $\Gamma$ is the full width at half maximum, and $q$ is the asymmetry parameter. The ftting of $\mathrm{TO}_{2}$ mode is shown as the insert of f gure 1 , and the phonon frequency is $170.2 \mathrm{~cm}^{-1}$.

In order to measure the radiation-induced lattice strain, the samples were characterized using single crystal x-ray diffraction in a symmetric scan mode. Figure 2(a) shows the (002) Bragg peak of $\mathrm{SrTiO}_{3}$ after $12 \mathrm{MeV}$ Ti ion irradiation to a fuence of $8.3 \times 10^{13}$ ions $\mathrm{cm}^{-2}$. The $\mathrm{x}$-ray beam penetrates the whole damaged layer, and the sharp peak in the high angle side is the Bragg peak from the undamaged part. The broad signal with lower $2 \theta$ angle comes from the damaged layers. The fringe features indicate the presence of a damage-induced dilation gradient in the direction normal to the sample surface [30]. The simulation of the diffraction pattern allows us to derive the depth-strain prof le. As shown by the insert in f gure 2(a), the derived strain prof le yields a good $\mathrm{ft}$ to the measured XRD pattern. The maximum strain occurs at depth of $\sim 3000 \mathrm{~nm}$, which is in good agreement with the predicted damage profle based on SRIM calculations [31].The lattice expansion along to01i from ion irradiation has a maximum out-of-plan strain of $1.32 \%$. Unlike thin flms, it is usually observed that there is no in-plane strain for ion-irradiated crystals [32]. In order to determine the in-plane lattice strain, reciprocal space maps (RSMs) were measured for both the symmetric (002) and asymmetric (103) Bragg peak (f gures 2(b) and (c)). The RSM of the asymmetric (103) peak shows only damage perpendicular to the sample surface. The scattering from both the damaged and undamaged region exhibit an identical value of $Q_{x}$ coordinate which demonstrates that the lattice expansion parallel to the surface is negligible. The lattice strain can also be estimated from the RSMs. The maximum lattice strain for the sample irradiated with $12 \mathrm{MeV}$ Ti ions is $1.31 \%$ and $1.30 \%$, as estimated from the (002) and (103) RSMs, respectively. The maximum lattice strain estimated from RSMs is in good agreement with the one-dimensional XRD measurement. Similar measurements were performed for the sample irradiated with $20 \mathrm{MeV}$ Ti ions. The XRD prof le around the (002) Bragg peak shows the damage peak without well-resolved fringes which forbids a reliable simulation. The RSMs around (002) and (103) Bragg peaks are shown in f gures 2(d) and (e), respectively. With a similar analysis, the maximum lattice strain in the sample irradiated with $20 \mathrm{MeV}$ Ti ions is estimated to be $1.13 \%$ from both (002) and (103) RSMs. As shown in the depth dependent strain prof le (f gure 2(a)), ion irradiation induced damage is not homogeneous along the path of the ions and, near the sample surface, the out-of-plane lattice strain is relatively weak $(<0.03 \%)$. It is thus important to check the structure in the whole damaged region. The maximum strain induced by $20 \mathrm{MeV}$ Ti ion irradiation is slightly less than that for $12 \mathrm{MeV}$ Ti ions, which indicates that the tetragonal distortion for $12 \mathrm{MeV} \mathrm{Ti}$ is larger than for $20 \mathrm{MeV} \mathrm{Ti}$, at least in the maximum damaged region. However, the Raman spectrum (f gure 1) clearly shows more ferroelectricity in the 20 $\mathrm{MeV}$ ion irradiated sample. This inconsistency suggested that symmetry breaking is not the only reason for the ferroelectric transition in ion irradiated $\mathrm{SrTiO}_{3}$ crystals.

Figure 3(a) shows the depth dependent Raman spectrum from the $\mathrm{SrTiO}_{3}$ sample irradiated with $20 \mathrm{MeV}$ Ti ions over the whole damaged depth $(<6 \mu \mathrm{m})$ with incremental depth measurement steps of $0.3 \mu \mathrm{m}$. The feature of ferroelectricity is clearly observed from the top to the maximum damaged depth $(\sim 3-4 \mu \mathrm{m})$. Beside the appearance of $\mathrm{TO}_{2}, \mathrm{TO}_{4}$ and $\mathrm{LO}_{4}$ modes, the second-order Raman modes shift the center positions at different depths. As shown by the dashed guidelines in f gure 3(a), the modes have red shifted gradually from surface to the maximum damaged depth of $\sim 3.9 \mu \mathrm{m}$, then quickly switched back to higher wavenumber values, as in the pristine sample. This is an indication of the inhomogeneous lattice expansion with depth, and the maximum damaged region has the minimum wavenumber because of the maximum lattice expansion. The positions of the new phonon modes are not sensitive to depth or damage levels. After the Fano ft, however, the frequency of the $\mathrm{TO}_{2}$ mode has undergone an observable shift $\left(<2 \mathrm{~cm}^{-1}\right)$ due to the change of peak shape. In the maximum damaged region $(2.4-3.3 \mu \mathrm{m})$, the peak position is nearly the same as the Fano ft (f gure 3(b)). The symmetric $\mathrm{TO}_{4}$ mode $\mathrm{ftted}$ with a Gaussian function shows no signif cant shift $\left(<1 \mathrm{~cm}^{-1}\right)$ from surface to the maximum damaged 

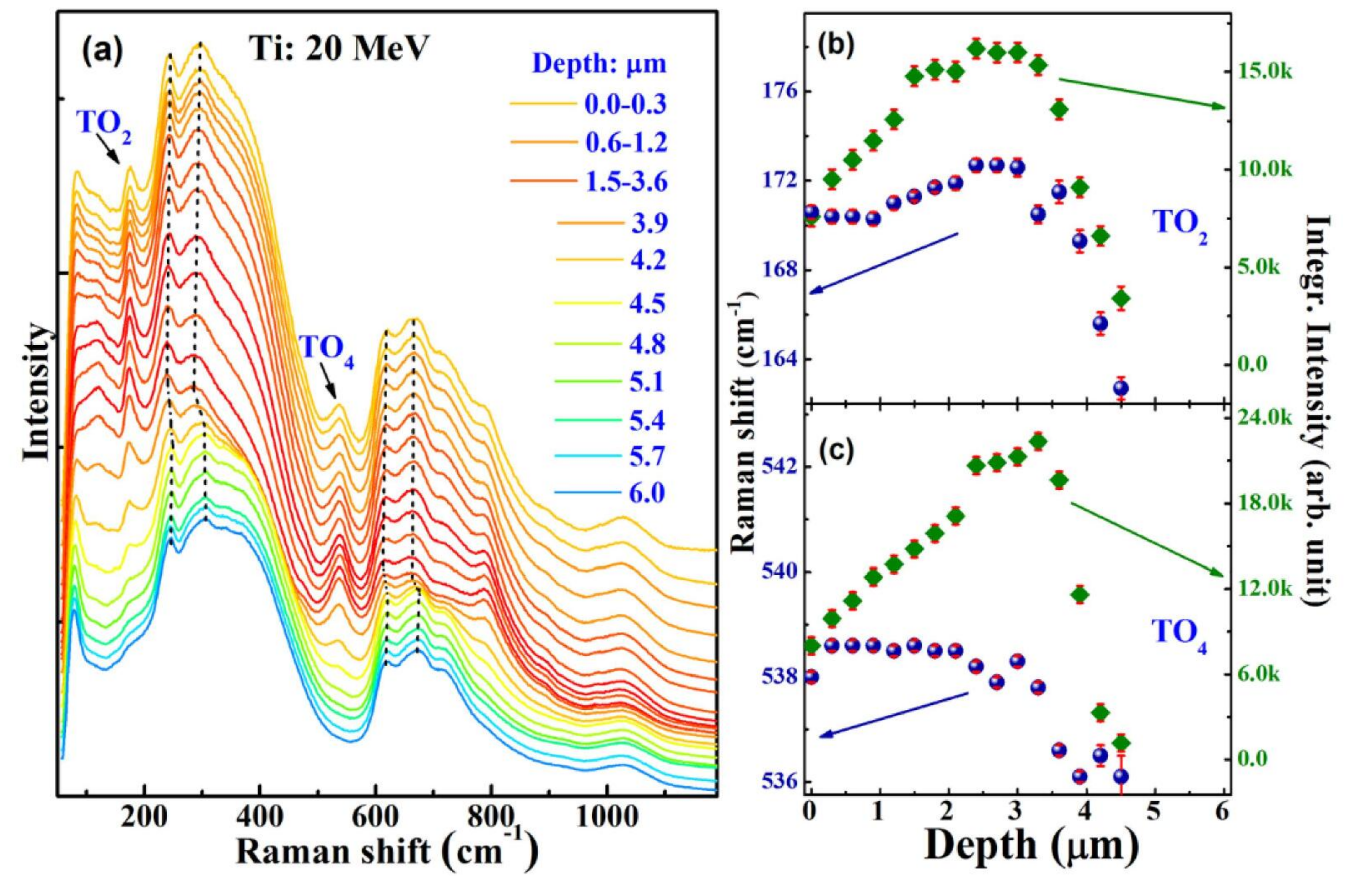

Figure 3. (a) The depth dependent Raman spectrum of $\mathrm{SrTiO}_{3}$ irradiated with $20 \mathrm{MeV}$ Ti ions. (b) and (c) The wavenumber and peak intensity of $\mathrm{TO}_{2}$ mode with Fano asymmetry $\mathrm{ftting}$ and $\mathrm{TO}_{4}$ mode with Gaussian $\mathrm{f}$ tting respectively.

region (f gure 3(c)). The intensity of both $\mathrm{TO}_{2}$ and $\mathrm{TO}_{4}$ modes increase with depth from the surface to the maximum damaged depth and then drops dramatically and eventually disappear. The cross-sectional Raman spectrum clearly indicates that the ferroelectricity starts from the very top surface to the maximum damaged region. The maximum damaged region has a tetragonal character, which can be seen from the weak tetragonal modes centered at $140 \mathrm{~cm}^{-1}$ and $478 \mathrm{~cm}^{-1}$, respectively. After the maximum damage, the intensity of all the new modes dropped rapidly, this is consistent with the damage prediction by SRIM, as discussed later. The depth dependent Raman spectrum also suggests that symmetry degeneration in the maximum damaged region is not the only reason of the ferroelectric transition in $\mathrm{SrTiO}_{3}$ crystal.

Raman spectrum (f gure 1) clearly indicates ferroelectric transition in $\mathrm{SrTiO}_{3}$ is dependent on the ion energies, and the ferroelectric feature of $20 \mathrm{MeV} \mathrm{Ti}$ ion irradiated sample is more obvious than that in $12 \mathrm{MeV}$ Ti ion irradiated samples. Figure 4 illustrates the energy deposition and damage levels (displacement per atom) for $\mathrm{SrTiO}_{3}$ irradiated with $20 \mathrm{MeV}$ and $12 \mathrm{MeV}$ Ti ions at room temperature based on full-cascade SRIM calculations [33]. Except for the different ion penetration depths, the damage levels for $20 \mathrm{MeV}$ and $12 \mathrm{Mev} \mathrm{Ti}$ ion irradiation of $\mathrm{SrTiO}_{3}$ are similar $(\sim 0.05 \mathrm{dpa})$. The elastic energy loss to atomic nuclei in $\mathrm{SrTiO}_{3}$ is very small $(<0.1$ $\mathrm{keV} \mathrm{nm}{ }^{-1}$ ) in the frst $1 \mu \mathrm{m}$ region and $<0.7 \mathrm{keV} \mathrm{nm}^{-1}$ in the maximum damage region. The much larger energy loss to the electrons is an inelastic process, and the effect on the lattice damage is not well understood [34]. Previous studies have shown that it has a complicated effect on the lattice, it can generate defects and, in the meanwhile, the strong electron-phonon coupling can also cause damage recovery, like

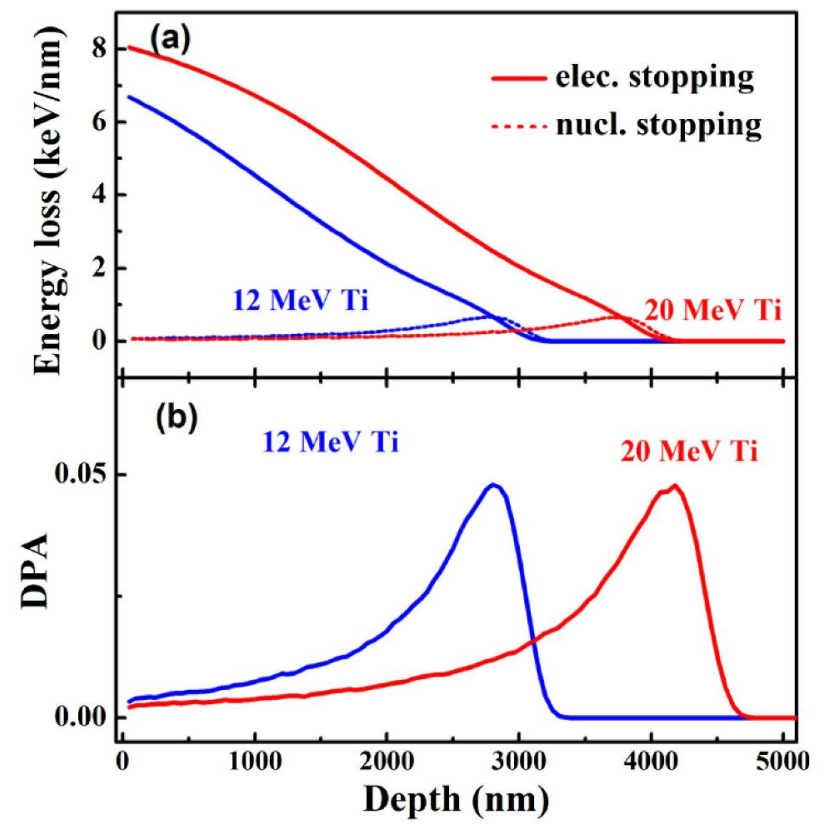

Figure 4. The calculated energy loss (a) and damage (b) in $\mathrm{SrTiO}_{3}$ crystal irradiated with $12 \mathrm{MeV}$ and $20 \mathrm{MeV}$ Ti ions.

for $\mathrm{MeV}$ ions irradiated $\mathrm{SiC}$ [35]. A synergistic effect of electronic energy loss with pre-existing defects in $\mathrm{SrTiO}_{3}$ has been conf rmed experimentally and theoretically $[34,36]$, and amorphous tracks and additional defects are generated in the predamaged crystal samples. High electron energy loss induces a localized thermal spike that can cause local melting of the sample along He ion path to form amorphous tracks via rapid quenching. The threshold in electron energy loss for forming 


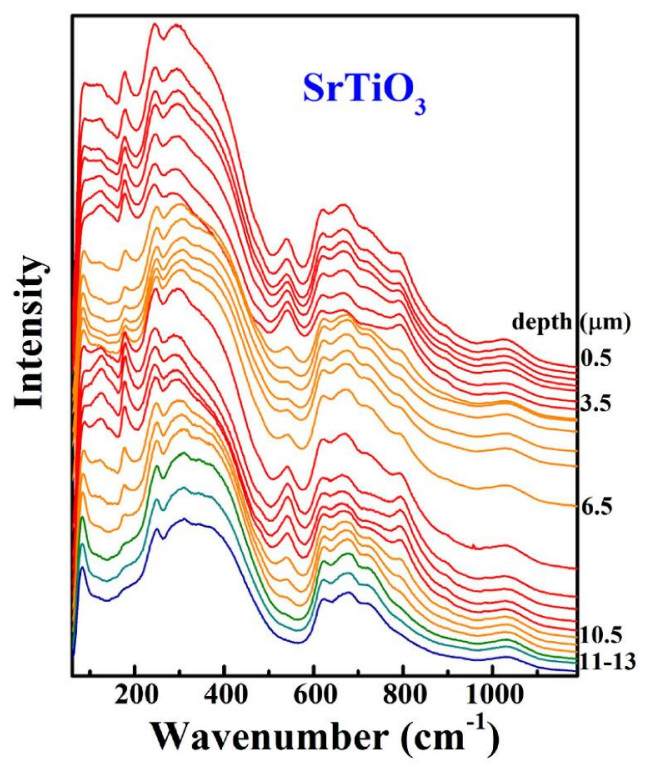

Figure 5. Cross section Raman spectrum of $\mathrm{SrTiO}_{3}$ irradiated with $20 \mathrm{MeV}$ Ti ions in fuence of $1 \times 10^{14} \mathrm{~cm}^{-2}$, and then measured with RBS, which the sample was bombard with $3.5 \mathrm{MeV}$ He ions. The ferroelectricity layer has a thickness more than $10 \mu \mathrm{m}$, and the step of depth is $0.5 \mu \mathrm{m}$ before $10.5 \mu \mathrm{m}$ and $1 \mu \mathrm{m}$ after $11 \mu \mathrm{m}$.

tracks in pristine $\mathrm{SrTiO}_{3}$ is $\sim 12.7 \mathrm{keV} \mathrm{nm}{ }^{-1}$ [37]; however, in the case of slightly pre-damaged $\mathrm{SrTiO}_{3}$, the threshold for amorphous track formation decreases signif cantly to about 6.5 $\mathrm{keV} \mathrm{nm}^{-1}[36,38]$. While the electron energy loss of $12 \mathrm{MeV}$ and $20 \mathrm{MeV}$ Ti ions is less than the threshold for amorphous track formation in pristine $\mathrm{SrTiO}_{3}$, their electronic energy loss is above the threshold for amorphous track formation in the presence of pre-existing defects. Thus, a delayed formation of amorphous tracks may occur once a suff cient concentration of defects accumulate due to nuclear collision processes, as has been observed in $\mathrm{LiTaO}_{3}$ [39]. Since the electronic energy loss for $20 \mathrm{MeV}$ Ti ions (f gure 4(b)) is obviously higher than that for $12 \mathrm{MeV}$ Ti ions, more damage production in the form of amorphous tracks and defects is expected for $20 \mathrm{MeV}$ Ti ions. The damage due to the electronic energy loss may be directly related to the ferroelectric transition in $\mathrm{SrTiO}_{3}$ crystals.

Room temperature ferroelectricity has been reported in non-stoichiometric $\mathrm{SrTiO}_{3}$ thin $\mathrm{flms}$ by controlling the oxygen def ciencies [19, 34]. Kim [20] has proposed the formation of a defect dipole by $\mathrm{Sr}-\mathrm{O}-\mathrm{O}$ clusters, which may be the possible origin of room temperature ferroelectricity in nonstoichiometric $\mathrm{SrTiO}_{3} \mathrm{flms}$. In addition to the tendency of a tetragonal structural transition, the room temperature ferroelectricity in $\mathrm{SrTiO}_{3}$ due to $\mathrm{Ti}$ ion irradiation may have a similar origin, because oxygen vacancies can be easily generated by $\mathrm{MeV}$ ion irradiation. Previous photoluminescence and $\mathrm{x}$-ray absorption measurements $[41,42]$ have suggested that ion irradiation of $\mathrm{SrTiO}_{3}$ readily yields oxygen vacancy disorder and near-surface irradiation of $\mathrm{SrTiO}_{3}$ with $\mathrm{Ar}$ ions also creates an oxygen def ciency [43].

Compared to thin $\mathrm{f} l \mathrm{~m}$ techniques, ion irradiation can make room temperature ferroelectricity in $\mathrm{SrTiO}_{3}$ over much larger thicknesses. Figure 5 shows the depth dependent Raman spectrum from $\mathrm{SrTiO}_{3}$ irradiated with $20 \mathrm{MeV}$ Ti ions to a dose of $1 \times 10^{14} \mathrm{~cm}^{-2}$, and then subsequently irradiated with 3.5 $\mathrm{MeV} \mathrm{He}$ ions for Rutherford backscattering spectroscopy measurements. The ferroelectricity is observed to a depth of $\sim 10$ $\mu \mathrm{m}$. Two maxima in the damaged regions due to $20 \mathrm{MeV}$ Ti ions and $3.5 \mathrm{MeV} \mathrm{He}$ ions irradiation are clearly seen from the Raman spectra. By irradiating with ions of high energies, such as swift heavy ions, it is expected that much thicker or even bulk $\mathrm{SrTiO}_{3}$ can be transformed to ferroelectric state at room temperature.

\section{Conclusions}

Room temperature ferroelectricity was detected by Raman scattering in $\mathrm{SrTiO}_{3}$ irradiated with Ti ions. X-ray diffraction measurements suggest that the transition is due to symmetry breaking, which caused by the large out-of-plane strains. $\mathrm{SrTiO}_{3}$ irradiated with $20 \mathrm{MeV}$ Ti ions shows more profound transition features than the sample irradiated with $12 \mathrm{MeV}$ Ti ions. Ion irradiation induced damage, especially the formation of oxygen vacancies, may play an important role for the ferroelectric transition in $\mathrm{SrTiO}_{3}$.

\section{Acknowledgments}

FZ would like to acknowledge the help from Dr Aurelien Debelle for the $\mathrm{x}$-ray measurements and strain analysis. This work was supported by the U.S. Department of Energy, Off ce of Science, Basic Energy of Sciences, Materials Sciences and Engineering Division under Contract DE-AC05-00OR22725. HX was supported by the University of Tennessee Governor's Chair program for RBS/C. The lab X-ray experiment was conducted at the Center for Nanophase Materials Sciences, which is a DOE Off ce of Science User Facility.

\section{References}

[1] Rimai L and Demars G A 1962 Electron paramagnetic resonance of trivalent gadolinium ions in strontium and barium titanates Phys. Rev. 127 702-10

[2] Weaver H E 1959 Dielectric properties of single crystals of $\mathrm{SrTiO}_{3}$ at low temperatures J. Phys. Chem. Solids 11 $274-77$

[3] Müller K A and Burkard $\mathrm{H} 1979 \mathrm{SrTiO}_{3}$ : an intrinsic quantum paraelectric below 4 K Phys. Rev. B $193593-602$

[4] Fujishita H, Kitazawa S, Saito M, Ishisaka R, Okamoto H and Yamaguchi T 2016 Quantum paraelectric states in $\mathrm{SrTiO}_{3}$ and $\mathrm{KTaO}_{3}$ : Barrett model, Vendik model, and quantum criticality J. Phys. Soc. Jpn. 85074703

[5] Haeni $\mathbf{J} \mathrm{H}$ et al 2004 Room-temperature ferroelectricity in strained $\mathrm{SrTiO}_{3}$ Nature $430758-61$ 
[6] Uwe H and Sakudo T 1976 Stress-induced ferroelectricity and soft phonon mode in $\mathrm{SrTiO}_{3}$ Phys. Rev. B 13271

[7] Ouillon R, Pinan-Lucarre J-P, Ranson P, Pruzan P, Mishra S K, Ranjan R and Pandey D 2002 A Raman scattering study of the phase transitions in $\mathrm{SrTiO}_{3}$ and in the mixed system $\left(\mathrm{Sr}_{1-x} \mathrm{Ca}_{x}\right) \mathrm{TiO}_{3}$ at ambient pressure from $T=300 \mathrm{~K}$ down to 8 K J. Phys.: Condens. Matter 14 2079-92

[8] Bianchi U, Kleemann W and Bednorz J G 1994 Raman scattering investigation of $\mathrm{Y}_{1-x} \mathrm{Ca}_{x} \mathrm{TiO}_{3} J$. Phys.: Condens. Matter 6 1229-38

[9] Itoh M, Wang R, Inaguma $\mathrm{Y}$, Yamaguchi $\mathrm{T}$, Shan $\mathrm{Y} J$ and Nakamura T 1999 Ferroelectricity induced by oxygen isotope exchange in strontium titanate perovskite Phys. Rev. Lett. 82 3540-3

[10] Fleury P A, Scott J F and Worlock J M 1968 Soft phonon modes and the $110 \mathrm{~K}$ phase transition in $\mathrm{SrTiO}_{3}$ Phys. Rev. Lett. 21 $16-9$

[11] Worlock J M and Fleury P A 1967 Electric feld dependence of optical-phonon frequencies Phys. Rev. Lett. 19 1176-9

[12] Banerjee S, Kim D I, Robinson R D, Herman I P, Mao Y and Wong S S 2006 Observation of Fano asymmetry in Raman spectra of $\mathrm{SrTiO}_{3}$ and $\mathrm{Ca}_{x} \mathrm{Sr}_{1-x} \mathrm{TiO}_{3}$ perovskite nanocubes Appl. Phys. Lett. 89223130

[13] Pai Y, Tylan-Tyler A, Irvin P and Levy J 2018 Physics of $\mathrm{SrTiO}_{3}$-based heterostructures and nanostructures: a review Rep. Prog. Phys. 81036503

[14] Li Y L et al 2006 Phase transitions and domain structures in strained pseudocubic (100) $\mathrm{SrTiO}_{3}$ thin $\mathrm{f}$ lms Phys. Rev. B 73 183112

[15] Jang H W et al 2010 Ferroelectricity in strain-free $\mathrm{SrTiO}_{3}$ thin flms Phys. Rev. Lett. 104197601

[16] Akimov I A, Sirenko A A, Clark A M, Hao J H and Xi X X 2000 Electric-f eld-induced soft-mode hardening in $\mathrm{SrTiO}_{3}$ Films Phys. Rev. Lett. 84 4625-8

[17] Sirenko A A, Bernhard C, Golnik A, Clark A M, Hao J, Si W and Xi X X 2000 Soft-mode hardening in $\mathrm{SrTiO}_{3}$ thin $\mathrm{f} 1 \mathrm{~ms}$ Nature $404373-6$

[18] Sirenko A A, Akimov I A, Fox J R, Clark A M, Li H C, Si W and Xi X X 1999 Observation of the frst-order Raman scattering in $\mathrm{SrTiO}_{3}$ thin $\mathrm{f}$ lms Phys. Rev. Lett. 82 4500-3

[19] Yang F, Zhang Q, Yang Z, Gu J, Liang Y, Li W, Wang W, Jin K, Gu L and Guo J 2015 Room-temperature ferroelectricity of $\mathrm{SrTiO}_{3}$ f lms modulated by cation concentration Appl. Phys. Lett. 107082904

[20] Kim Y S, Kim J, Moon S J, Choi W S, Chang Y J, Yoon J G, Yu J, Chung J S and Noh T W 2009 Localized electronic states induced by defects and possible origin of ferroelectricity in strontium titanate thin flms Appl. Phys. Lett. 94202906

[21] Choi M, Oba F and Tanaka I 2009 Role of Ti antisitelike defects in $\mathrm{SrTiO}_{3}$ Phys. Rev. Lett. 103185502

[22] Cowley R A 1964 Lattice dynamics and phase transitions of strontium titanate Phys. Rev. 134 A981-7

[23] Tenne D A et al 2016 Probing nanoscale ferroelectricity by ultraviolet Raman spectroscopy Science 313 1614-6

[24] Zhang Y, Crespillo M, Xue H, Jin K, Chen C H, Fontana C L, Graham J T and Weber W J 2014 New ion beam materials laboratory for materials modif cation and irradiation effects research Nucl. Instrum. Methods Phys. Res. B 338 19-30

[25] Souilah M, Boulle A and Debelle A 2016 Computer programs RaDMaX: a graphical program for the determination of strain and damage prof les in irradiated crystals J. Appl. Crystallogr. $49311-6$
[26] Rychetský I et al 2001 Dielectric, infrared, and Raman response of undoped $\mathrm{SrTiO}_{3}$ ceramics: evidence of polar grain boundaries Phys. Rev. B 64184111

[27] Ostapchuk T et al 2002 Origin of soft-mode stiffening and reduced dielectric response in $\mathrm{SrTiO}_{3}$ thin flms Phys. Rev. B 66235406

[28] Perry C H, Fertel J H and McNelly T F 1967 Temperature dependence of the Raman spectrum of $\mathrm{SrTiO}_{3}$ and $\mathrm{KTaO}_{3} \mathrm{~J}$. Chem. Phys. 47 1619-25

[29] Fano U 1961 Effects of integration on intensities and phase shifts Phys. Rev. 124 1866-78

[30] Thomé L, Velisa G, Miro S, Debelle A, Garrido F, Sattonnay G, Mylonas S, Trocellier P and Serruys Y 2015 Recovery effects due to the interaction between nuclear and electronic energy losses in $\mathrm{SiC}$ irradiated with a dual-ion beam J. Appl. Phys. 117105901

[31] Ziegler J F 2004 SRIM-2003 Nucl. Instrum. Methods Phys. Res. B 219-220 1027-36

[32] Debelle A and Declémy A 2010 XRD investigation of the strain/stress state of ion-irradiated crystals Nucl. Instrum. Methods Phys. Res. B 268 1460-5

[33] Weber W J and Zhang Y 2019 Predicting damage production in monoatomic and multi-elemental targets using stopping and range of ions in matter code: challenges and recommendations Curr. Opin. Solid State Mater. Sci. 23100757

[34] Weber W J, Zarkadoula E, Pakarinen O H, Sachan R, Chisholm M F, Liu P, Xue H, Jin K and Zhang Y 2015 Synergy of elastic and inelastic energy loss on ion track formation in $\mathrm{SrTiO}_{3} \mathrm{Sci}$. Rep. 57726

[35] Zhang Y, Sachan R, Pakarinen O H, Chisholm M F, Liu P, Xue H and Weber W J 2015 Ionization-induced annealing of pre-existing defects in silicon carbide Nat. Commun. 6 8049

[36] Xue H, Zarkadoula E, Liu P, Jin K, Zhang Y and Weber W J 2017 Amorphization due to electronic energy deposition in defective strontium titanate Acta Mater: 127 400-6

[37] Karlusic M, Akcoltekin S, Osmani O, Monnet I, Lebius H, Jaksic M and Schleberger M 2010 Energy threshold for the creation of nanodots on $\mathrm{SrTiO}_{3}$ by swift heavy ions New J. Phys. 12043009

[38] Xue H, Zarkadoula E, Sachan R, Zhang Y, Trautmann C and Weber W J 2018 Synergistically-enhanced ion track formation in pre-damaged strontium titanate by energetic heavy ions Acta Mater. 150 351-9

[39] Sellami N, Crespillo M L, Zhang Y and Weber W J 2018 Twostage synergy of electronic energy loss with defects in $\mathrm{LiTaO}_{3}$ under ion irradiation Mater. Res. Lett. $6339-44$

[40] Lee S A et al 2016 Phase transitions via selective elemental vacancy engineering in complex oxide thin $\mathrm{flms} S \mathrm{Sci}$. Rep. 6 23649

[41] Crespillo M L, Graham J T, Agulló-López F, Zhang Y and Weber W J 2017 Correlation between $\mathrm{Cr}^{3+}$ luminescence and oxygen vacancy disorder in strontium titanate under $\mathrm{MeV}$ ion irradiation J. Phys. Chem. C 121 19758-66

[42] Sabathier C, Chaumont J, Rouzière S and Traverse A 2005 Characterisation of Ti and $\mathrm{Sr}$ atomic environments in $\mathrm{SrTiO}_{3}$ before and after ion beam irradiation by $\mathrm{x}$-ray absorption spectroscopy Nucl. Instrum. Methods Phys. Res. B 234 $509-19$

[43] Kan D, Terashima T, Kanda R, Masuno A, Tanaka K, Chu S, Kan H, Ishizumi A, Kanemitsu Y, Shimakawa Y and Takano M 2005 Blue-light emission at room temperature from $\mathrm{Ar}^{+}$irradiated $\mathrm{SrTiO}_{3}$ Nat. Mater. 4 816-9 